\section{Postpartum infective endocarditis with Enterococcus faecalis after vaginal delivery}

\author{
İbrahim Etem Dural* (D), Serkan Gökaslan* (D), Zafer Yalım* (D), Uğur Aksı* (D), \\ Ömer Faruk Yılmaz* (D) İbrahim Ersoy* (D), Fehim Can Sevil** (D) \\ Departments of *Cardiology, and **Cardiovascular Surgery, Afyonkarahisar \\ University of Health Sciences; Afyonkarahisar-Turkey
}

\section{Introduction}

Infective endocarditis (IE) is rare in the young population and has a high mortality rate. Traditional risk factors such as valve replacement, cardiac device implantations, and continuous intravenous drug use are well defined in IE. IE after urogenital surgery is less common than dental or gastrointestinal procedures (1). In addition, the pathogens causing endocarditis and the location of cardiac involvement may vary depending on the type of risk factors. Enterococci in the urogenital tract flora can cause about $10 \%$ of all IE.

Although the agents in pregnancy and postpartum IE are often staphylococci and streptococci, enterococci can also be causative agents, especially after a cesarean section (2). IE with enterococci after vaginal delivery is very rare. Here, we present postpartum IE with Enterococcus faecalis in a 24-year-old healthy woman. To the best of our knowledge, the cases of IE with Enterococcus faecalis after vaginal delivery are extremely rare in the literature.

\section{Case Report}

A 24-year-old female patient without a known history of cardiac disease visited our clinic with complaints of dyspnea, fever, and cough. According to her medical history, she did not take any intravenous drugs and did not travel anywhere for two years. She had a healthy baby with vaginal delivery 7 months ago. Fatigue and dyspnea with exertion started two weeks after delivery, and the dyspnea progressed with fever starting in the past week.

Physical examination revealed tachypnea and tachycardia with a $3 / 6$ diastolic murmur in the mitral region. Her body temperature was $38.5^{\circ} \mathrm{C}$, respiratory rate $30 \mathrm{bpm}$, heart rate 115 bpm, and blood pressure 110/70 $\mathrm{mm} \mathrm{Hg}$.

Coronavirus disease 2019 infection was ruled out with a negative thorax computed tomography scan. Valvular heart disease was suspected, and a transthoracic echocardiogram (TTE) was performed to evaluate cardiac structures and functions. Three sets of blood cultures were drawn on admission.

TTE showed an ejection fraction of $55 \%$, severe mitral insufficiency, a mass compatible with vegetation on both the anterior and posterior leaflets of the mitral valve. In addition, ruptured chordae tendineae was detected.

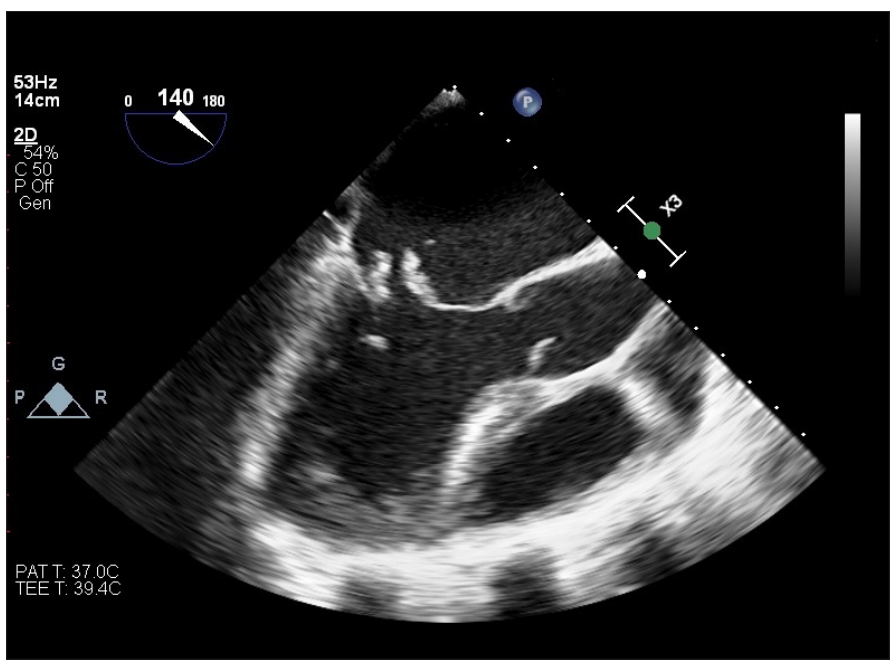

Figure 1. Two-dimensional transesophageal echocardiography showing a vegetation-like mass on the anterior and posterior leaflets of the mitral valve and ruptured chordae tendineae

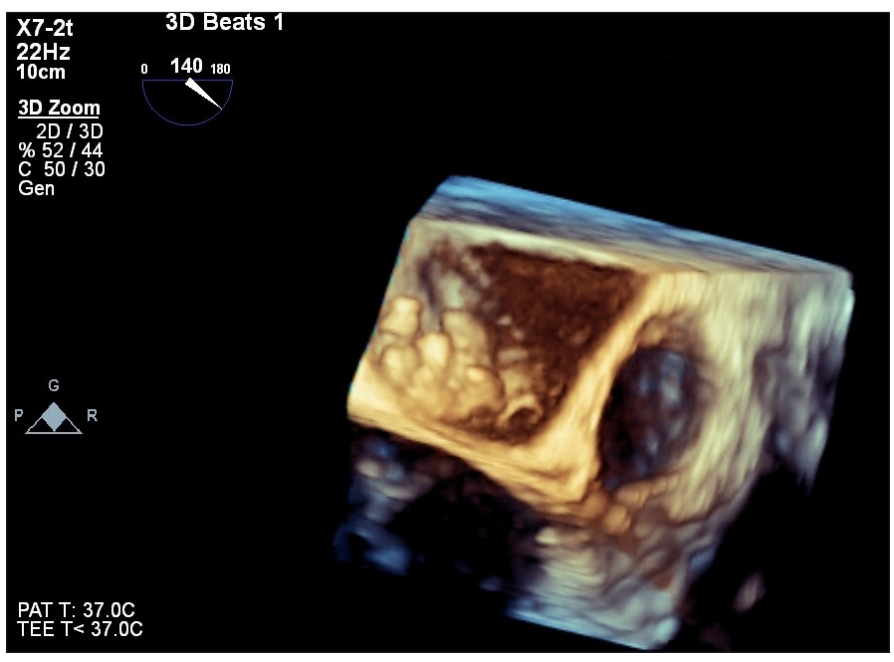

Figure 2. Three-dimensional transesophageal echocardiography showing a vegetation-like mass on the anterior and posterior leaflets of the mitral valve and ruptured chordae tendineae

After echocardiographic evaluation, IE was diagnosed, and empiric antibiotic therapy was administered (vancomycin $500 \mathrm{mg}$ once a day and gentamicin $80 \mathrm{mg}$ twice a day). Transesophageal echocardiogram (TEE) revealed a mobile $20 \times 18 \mathrm{~mm}$ vegetation attached to the anterior mitral valve, and a mobile $14 \times 11 \mathrm{~mm}$ vegetation attached to the posterior mitral valve. Chordae tendineae rupture was observed in both anterior and posterior leaflets. Severe mitral regurgitation was observed in TEE (Fig. 1, 2 and Videos 1, 2). On the third day of her hospitalization, blood culture results were positive for vancomycin resistant Enterococcus faecalis, and daptomycin treatment was initiated.

She did not have fever on the fourth day of her hospitalization. Her complaints of dyspnea and cough regressed. The patient underwent mitral valve replacement operation on the $14^{\text {th }}$ day of hospitalization. No vegetation or positive blood cul- 
ture was detected during the follow-up period. She was discharged on the $42^{\text {nd }}$ day of hospitalization.

\section{Discussion}

IE in the postpartum period is a very rare and life-threatening clinical condition. Suppressed immunity during pregnancy and the postpartum period and invasive procedures performed during delivery such as insertion of a urinary catheter may cause disruption of the mucosal barrier and as a result, local infections may occur. Local infections caused by opportunistic organisms can rarely cause systemic infections and sepsis, which can be life-threatening (3). Cesarean sections and routine urinary catheter insertions are particular risk factors for local infections. Therefore, many obstetrics clinics administer antibiotic prophylaxis before cesarean section (4) However, vaginal delivery without risk factors has a lower risk of infection, and routine prophylaxis is not recommended in the current guidelines (5). Our patient presented with a serious infection that developed after vaginal delivery. A urinary catheter was not inserted, and antibiotic prophylaxis was not administered to our patient during delivery, only an episiotomy was performed before delivery.

The publications on pregnancy and postpartum IE are limited to case reports and small studies. Staphylococci and streptococci are the most common organisms in pregnancy-related endocarditis as in all IE. Although IE with enterococci is known at $10 \%$ in the general population, this rate is lower in peripartum and postpartum endocarditis. In a study in which 90 peripartum infective endocarditis case reports were examined, the rate of endocarditis with enterococci was reported at $1.1 \%(2)$.

Enterococcus faecalis is a gram-positive, catalase-negative bacterium inhabiting the gastrointestinal tract of humans. A plasmid-encoded hemolysin, called the cytolysin, is important for pathogenesis in animal models of infection. It could cause endocarditis and sepsis, urinary tract infections, and meningitis. The most important feature of enterococci is partial or complete resistance to many antimicrobial agents used in the treatment of gram-positive bacterial infections. The frequency of enterococci infection in all types of nosocomial infections, especially urinary tract infections, is increasing.

When we searched for studies on IE with Enterococcus faecalis on Web of Science and Google Scholar, we found 2 cases in the postpartum period. One of these developed after a cesarean section, and the other one was IE involving the aortic valve $(6,7)$. To the best of our knowledge, our patient is first case of mitral valve IE caused by Enterococcus feacalis after a vaginal delivery in these indexes. She presented with large vegetation and ruptured chordae tendineae because the hospital admission was delayed. This case showed that IE can be seen in healthy women with vaginal delivery. In addition, this case demonstrated that patients with persistent fever, weakness, and dyspnea symptoms in the postpartum period require a cardiac examination. Routine antibiotic use after vaginal delivery and episiotomy is still debatable, and large-scale randomized controlled studies are needed for further delineation.

\section{Conclusion}

IE has a high mortality rate, and early diagnosis and immediate antibiotherapy are crucial. During the postpartum period, the patient's signs and symptoms should be evaluated carefully, and appropriate patients should be referred to be evaluated for cardiac function and structures.

Informed consent: Written informed consent was obtained from the patients for sharing their relevant medical history and laboratory results.

Video 1. Two-dimensional transesophageal echocardiography showing a vegetation-like mass on the anterior and posterior leaflets of the mitral valve and ruptured chordae tendineae

Video 2. Two-dimensional transesophageal echocardiography showing a vegetation-like mass on the anterior and posterior leaflets of the mitral valve, ruptured chordae tendineae, and severe mitral regurgitation

\section{References}

1. Baddour LM, Wilson WR, Bayer AS, Fowler VG Jr, Tleyjeh IM, Rybak MJ, et al.; American Heart Association Committee on Rheumatic Fever, Endocarditis, and Kawasaki Disease of the Council on Cardiovascular Disease in the Young, Council on Clinical Cardiology, Council on Cardiovascular Surgery and Anesthesia, and Stroke Council. Infective Endocarditis in Adults: Diagnosis, Antimicrobial Therapy, and Management of Complications: A Scientific Statement for Healthcare Professionals From the American Heart Association. Circulation 2015; 132: 143586. [Crossref]

2. Kebed KY, Bishu K, Al Adham RI, Baddour LM, Connolly HM, Sohail $M R$, et al. Pregnancy and postpartum infective endocarditis: a systematic review. Mayo Clin Proc 2014; 89: 1143-52. [Crossref]

3. Phillips C, Walsh E. Group A Streptococcal Infection During Pregnancy and the Postpartum Period. Nurs Womens Health 2020; 24: 13-23. [Crossref]

4. Saeed KB, Corcoran P, Greene RA. Incisional surgical site infection following cesarean section: A national retrospective cohort study. Eur J Obstet Gynecol Reprod Biol 2019; 240: 256-60. [Crossref]

5. Sharma S, Dhakal I. Cesarean vs Vaginal Delivery : An Institutional Experience. JNMA J Nepal Med Assoc 2018; 56: 535-9. [Crossref]

6. Gopaul K, Padilla-Lopez C, Azeem A, Cherazard R. Post partum infective endocarditis caused by $\mathrm{E}$. faecium without predisposing disease. Abstract published at Hospital Medicine 2019, March 24-27, National Harbor, Md. Abstract 688.

7. Tamura M, Shoji M, Fujita K, Nakamura S, Takahashi Y, Suzuki Y, Asakura M, Kimizuka S, Sasaki M, Sugawara K. Postpartum infective endocarditis with Enterococcus faecalis in Japan: a case report. J Med Case Rep 2017; 11: 324. [Crossref]

Address for Correspondence: Dr. Ibrahim Etem Dural,

Afyonkarahisar Sağlık Bilimleri Üniversitesi,

Kardiyoloji Anabilim Dalı, Afyonkarahisar-Türkiye

Phone: +90 5544813137

E-mail: iedural@hotmail.com

CCopyright 2021 by Turkish Society of Cardiology -

Available online at www.anatoljcardiol.com

DOI:10.5152/AnatolJCardiol.2021.29797 